\title{
BIDUALS OF BANACH SPACES WITH BASES
}

\author{
by STEVEN F. BELLENOT
}

(Received 17 January, 1984)

R. C. James [2] (or see p. 7ff of [3]) gave a useful representation of the bidual of any space with a shrinking basis. This note gives a representation of the bidual of any space with a basis.

Our notation follows that of [3], where undefined terms can be found. Let $\left\{e_{n}\right\}$ be a basic sequence with coefficient functionals $\left\{f_{n}\right\}$. We will assume $\left\{e_{n}\right\}$ is bimonotone; that is $\left\|\sum_{N}^{M} a_{n} e_{n}\right\| \leqslant\left\|\sum a_{n} e_{n}\right\|$. The space $\left\{e_{n}\right\}^{\mathrm{LIM}}$ is the set of scalar sequences $\left\{a_{n}\right\}$ so that $\left\|\left\{a_{n}\right\}\right\|=$ $\sup \left\|\sum_{1}^{N} a_{n} e_{n}\right\|<\infty$. We will abuse notation and equate such $\left\{a_{n}\right\}$ with the formal sum $\sum a_{n} e_{n}$. We have $\left[e_{n}\right]^{*}$ is $\left\{f_{n}\right\}^{\mathrm{LIM}}$. The basic sequence $\left\{e_{n}\right\}$ is boundedly complete if $\left\{e_{n}\right\}^{\mathrm{LIM}}=\left[e_{n}\right]$. The basic sequence $\left\{e_{n}\right\}$ is shrinking if $\left\{f_{n}\right\}$ is boundedly complete. James's result mentioned above is that for shrinking $\left\{e_{n}\right\}$, we have $\left[e_{n}\right]^{* *}=\left\{e_{n}\right\}^{\mathrm{LIM}}$.

A basic sequence $\left\{e_{n}\right\}$ is wild if there is $\left\{a_{n}\right\} \in\left\{e_{n}\right\}^{\mathrm{LIM}}$ and $\left\{b_{n}\right\} \in\left\{f_{n}\right\}^{\mathrm{LIM}}$ so that $\left\{\sum_{1}^{N} a_{n} b_{n}\right\}_{N}$ does not converge. Define $Z\left(e_{n}\right)$ to be the space $\left\{e_{n}\right\}^{\mathrm{LIM}} /\left[e_{n}\right]$ and let $Z^{*}\left(e_{n}\right)$ be $\phi^{*}\left(Z\left(e_{n}\right)^{*}\right)$, where $\phi$ is the induced quotient map.

If $\mathcal{U}$ is a free ultrafilter on $N$ (i.e. $\cap \mathcal{U}=\varnothing$ ) and $\left\{a_{n}\right\}$ is a bounded scalar sequence, then $\lim _{\mathcal{U}} a_{n}=L$ means that $\left\{n \in N:\left|a_{n}-L\right|<\varepsilon\right\} \in \mathcal{U}$ for all $\varepsilon>0$. Note that this limit must exist and there is a subsequence $\{n(i)\}$ such that $a_{n(i)} \rightarrow L$. Conversely, if $a_{n(i)} \rightarrow L$, then there is a free ultrafilter $U$ such that $\lim a_{n}=L$. Note that by using an ultrafilter instead of a subsequence we get convergence for all bounded $\left\{a_{n}\right\}$. Define $\sum_{\alpha_{L}} a_{n}$ to be $\lim _{\mathcal{L}} \sum_{1}^{n} a_{\mathrm{i}}$.

Theorem. Let $\mathcal{U}$ be a free ultrafilter on $N$. Define $T_{\ell}:\left\{e_{n}\right\}^{\mathrm{LIM}} \rightarrow\left[e_{n}\right]^{* *}=\left(\left\{f_{n}\right\}^{\mathrm{LIM}}\right)^{*}$ by

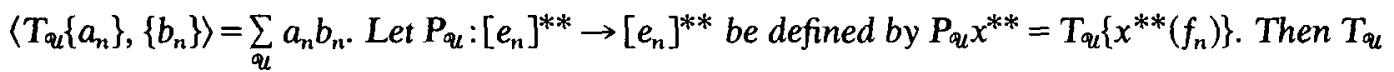
is an isometry, $\left\|P_{\text {qu}}\right\|=\left\|I-P_{q u}\right\|=1$ and $\left[e_{n}\right]^{* *}=T_{q u}\left(\left\{e_{n}\right\}^{\mathrm{LIM}}\right) \oplus Z^{*}\left(f_{n}\right)$.

Proof. Since $\left|\sum_{1}^{N} a_{n} b_{n}\right| \leqslant\left\|\sum_{1}^{N} a_{n} e_{n}\right\| \cdot\left\|\sum_{1}^{N} b_{n} f_{n}\right\| \leqslant\left\|\left\{a_{n}\right\}\right\| .\left\|\left\{b_{n}\right\}\right\|, \sum_{a_{u}} a_{n} b_{n}$ is well-defined. Thus $T_{u}\left\{a_{n}\right\}$ is a linear functional on $\left\{f_{n}\right\}^{\mathrm{LM}}$ and $\left\|T_{u}\left\{a_{n}\right\}\right\| \leqslant\left\|\left\{a_{n}\right\}\right\|$. Actually the norms are equal since if $b_{n}=0$ for $n \geqslant N$, then $\sum_{Q_{\ell}} a_{n} b_{n}=\sum_{1}^{N} a_{n} b_{n}$ and since $\left\{e_{n}\right\}^{\text {LIM }}$ is normed by $\left[f_{n}\right]$.

If $x^{* *} \in\left[e_{n}\right]^{* *}$ and $a_{n}=x^{* *}\left(f_{n}\right)$, then $\left\|\sum_{1}^{n} a_{i} e_{i}\right\|$ is equal to the norm of $x^{* *}$ restricted to

Glasgow Math. J. 26 (1985) 101-103. 
$\left[f_{i}\right]_{1}^{n}$ which is $\leqslant\left\|x^{* *}\right\|$. Hence $P_{\text {ou }}$ is a norm one projection onto $T_{u}\left(\left\{e_{n}\right\}^{\text {UM }}\right)$ with kernel $\left[f_{n}\right]^{\perp}=Z^{*}\left(f_{n}\right)$. Suppose that $z^{*} \in Z^{*}\left(f_{n}\right)$ with $\left\|z^{*}\right\|=1$ and $\left\{a_{n}\right\} \in\left\{e_{n}\right\}^{\mathrm{LM}}$; let $x^{* *}=$ $T_{u}\left\{a_{n}\right\}+z^{*}$. Let $z \in Z\left(f_{n}\right)$ so that $\|z\|=1$ and $z^{*}(z)>1-\varepsilon$. Pick $\left\{b_{n}\right\} \in\left\{f_{n}\right\}^{\mathrm{LIM}}$; so that $\left\|\sum b_{n} f_{n}\right\|<1+\varepsilon$ and $\phi\left(\sum b_{n} f_{n}\right)=z$. Choose $N$ so that $\left|\sum_{1}^{N} a_{n} b_{n}-\sum_{\vartheta_{u}} a_{n} b_{n}\right|<\varepsilon$ and thus $\left|\left\langle T_{\mathcal{u}}\left\{a_{n}\right\}, \sum_{N+1}^{\infty} b_{n} f_{n}\right\rangle\right|<\varepsilon$. Now $\phi\left(\sum_{N+1}^{\infty} b_{n} f_{n}\right)=z$ and the bimonotoneness condition implies $\left\|\sum_{N+1}^{\infty} b_{n} f_{n}\right\|<1+\varepsilon$. Hence $\left\|x^{* * *}\right\|>(1-2 \varepsilon) /(1+\varepsilon)$ and $\left\|I-P_{u}\right\|=1$.

Remarks. 1. Note that in general $\left\{e_{n}\right\}^{\mathrm{LM}}$ is just a quotient of $\left[e_{n}\right]^{* *}$, and since there are wild bases (see below) something like this ultrafilter sum is needed.

2. This is similar to embedding $\left[e_{n}\right]^{* *}$ in the nonstandard hull of $\left[e_{n}\right]$ and is where the author obtained the original proof.

3. If $\mathscr{U}(\alpha), \alpha \in \Gamma$, are different ultrafilters so that $\sum_{\mathcal{U}(\alpha)} a_{n} b_{n}, \alpha \in \Gamma$, are all distinct for some fixed $\left\{a_{n}\right\} \in\left\{e_{n}\right\}^{\mathrm{LIM}}$ and $\left\{b_{n}\right\} \in\left\{f_{n}\right\}^{\mathrm{LIM}}$, then for non-negative scalars $t_{\alpha}$ with $\sum_{\alpha} t_{\alpha}=1$ we have $\left\|\sum_{\alpha} t_{\alpha} T_{\mathcal{u ( \alpha )}}\left\{a_{n}\right\}\right\|=\left\|\left\{a_{n}\right\}\right\|$.

4. The Theorem immediately generalizes to spaces with a Schauder decomposition $[3$, p. $47 \mathrm{ff}]$ with all but finitely many of the factors being reflexive, but not infinitely many [1].

5. In light of [3, p. 26] or $[\mathbf{1}], Z^{*}\left(f_{n}\right)$ could be almost anything. No representation theorem can say much about this space.

EXAMPLE. We now exhibit a space with a wild basis. Let $X$ be a Banach space with a bimonotone basis $\left\{e_{n}\right\}$. Let $\left\{b_{n}\right\}$ be a bounded sequence and let $F$ be the "formal" function $\sum b_{n} f_{n}$. Let $\|.\|_{X}$ be the norm on $X$ and define

$$
\left\|\sum a_{n} e_{n}\right\|_{F}=\max \left\{\left\|\sum a_{n} e_{n}\right\|_{X}, \sup \left\{\left|\sum_{N}^{M} a_{n} b_{n}\right|: N \leqslant M\right\}\right\} .
$$

Let $Y=\left\{x \in X:\|x\|_{F}<\infty\right\}$ and note that $\left\{e_{n}\right\}$ is a bimonotone basis for $Y$ and that $F$ is a continuous linear functional on $Y$. Thus $X=Y$ if and only if $F \in\left\{f_{n}\right\}^{\mathrm{LM}}$.

If $X$ is $c_{0}$ and $b_{n}=(-1)^{n}$, then the resulting space $Y$ still has $\sum e_{n} \in\left\{e_{n}\right\}^{\mathrm{LIM}}$. Hence the basis $\left\{e_{n}\right\}$ is wild in $Y$. Let the set of even integers belong to $U$ and the set of odd integers belong to $\mathcal{U}^{\prime}$. Then $\left(T_{U_{U}}+T_{\mathscr{U}^{\prime}}\right) / 2$ is an isometry of $\left\{e_{n}\right\}^{\mathrm{LIM}}$ into $Y^{* *}$ which is not $T_{\mathcal{V}}$ for any ultrafilter $\mathcal{V}$.

If instead we choose $b_{n}$ to be the sequence $1,-1,2^{-1}, 2^{-1},-2^{-1},-2^{-1}, 2^{-2}, 2^{-2}, 2^{-2}$, $2^{-2},-2^{-2},-2^{-2},-2^{-2},-2^{-2}, \ldots$ then there are uncountably many ultrafilters $\mathcal{U}(\alpha)$ so that $T_{T_{U(\alpha)}}\left(\sum e_{n}\right) \neq T_{\left.\mathcal{U}_{(\beta)}\right)}\left(\sum e_{n}\right)$, for $\alpha \neq \beta$.

If $f \notin\left\{f_{n}\right\}^{\mathrm{LIM}}$, then the space $Y$ always has a subspace isomorphic to $c_{0}$. However, since being a wild basis is self-dual, this isn't true of all wild bases. 


\section{REFERENCES}

1. S. F. Bellenot, The $J$-sum of Banach spaces, $J$. Functional Analysis 48 (1982), 95-106.

2. R. C. James, Bases and reflexivity of Banach spaces, Ann. of Math. 52 (1950), 518-527.

3. J. Lindenstrauss and L. Tzafriri, Classical Banach spaces I Sequence Spaces (Springer Verlag, 1977).

Department of Mathematics and Computer Science

The Florida State University

TALlahassee

FLORIDA 32303

U.S.A. 